\title{
Abundances of $\mathrm{C}, \mathrm{N}$ and $\mathrm{O}$ as probes of mixing in low-mass helium-core burning stars
}

\author{
G. Tautvaišiené $\dot{1}^{1}$, B. Edvardsson ${ }^{2}$, E. Puzeras ${ }^{1}$ and I. Ilyin ${ }^{3}$ \\ ${ }^{1}$ Institute of Theoretical Physics and Astronomy of Vilnius University, \\ Gostauto 12, 01108 Vilnius, Lithuania \\ email: taut@itpa.lt \\ ${ }^{2}$ Department of Astronomy and Space Physics, Uppsala Observatory, \\ Box 515, 75120 Uppsala, Sweden \\ email: bengt.edvardsson@astro.uu.se \\ ${ }^{3}$ Astrophysical Institute Potsdam, An der Sternwarte 16, 14482 Potsdam, Germany \\ email: ilyin@aip.de
}

\begin{abstract}
A large population of helium-core-burning clump stars of the Galactic field, revealed by the Hipparcos orbiting observatory, is an excellent target for the analysis of mixing processes in evolved low-mass metal-abundant stars. In this contribution we overview first result of ${ }^{12} \mathrm{C}$, ${ }^{13} \mathrm{C}, \mathrm{N}$ and $\mathrm{O}$ abundance determinations in our extensive project which we started with the aim at deriving the fundamental parameters and abundances of more than 20 chemical elements in a large sample of Galactic clump stars.
\end{abstract}

Keywords. Stars: abundances, stars: horizontal-branch

\section{Introduction}

Low-mass stars exhibit signatures of extra-mixing processes that require modeling beyond the standard stellar theory and observational data for stars of different masses and metallicities. Hipparcos orbiting observatory has allowed to identify a large number of helium-core-burning "clump" stars of the Galactic field. They are excellent targets for the analysis of mixing processes in evolved low-mass metal-abundant stars.

A high-resolution spectroscopic analysis of 671 Galactic field metal abundant giants was made by McWilliam (1990). However, as it was pointed out later by McWilliam \& Rich (1994), the McWilliam (1990) study is hampered by the narrow wavelength $(6550-6800 \AA)$ coverage and the lack of metal-rich model atmospheres, which caused an underestimation of metallicities for metal rich stars. This was confirmed by Tautvaišiene et al. (2005). The mean difference of $[\mathrm{Fe} / \mathrm{H}]$ according to 35 common stars in these studies is $[\mathrm{Fe} / \mathrm{H}]_{M c W 90}-[\mathrm{Fe} / \mathrm{H}]_{T 05}=-0.13 \pm 0.07$. The effective temperature calibrations of McWilliam (1990) and of Alonso et al. (2001) have a slight systematic temperature determination difference of $54 \pm 50 \mathrm{~K}$ as well. A study of 39 red clump giants was accomplished by Zhao et al. (2001). Unfortunately, the determination of effective temperatures in this work was done using the erroneous temperature calibration by Alonso et al. (1999), which was afterwords corrected by Alonso et al. (2001).

The previous studies of the Galactic metal-rich clump stars do not contain detailed and homogeneous determinations of carbon and nitrogen abundances. This fact impelled us to start a comprehensive high-resolution spectral analysis of clump stars of the Galaxy. Our results of the main atmospheric parameter determinations for 45 clump stars are discussed in the contribution by Tautvaišienè et al. (2005a). 


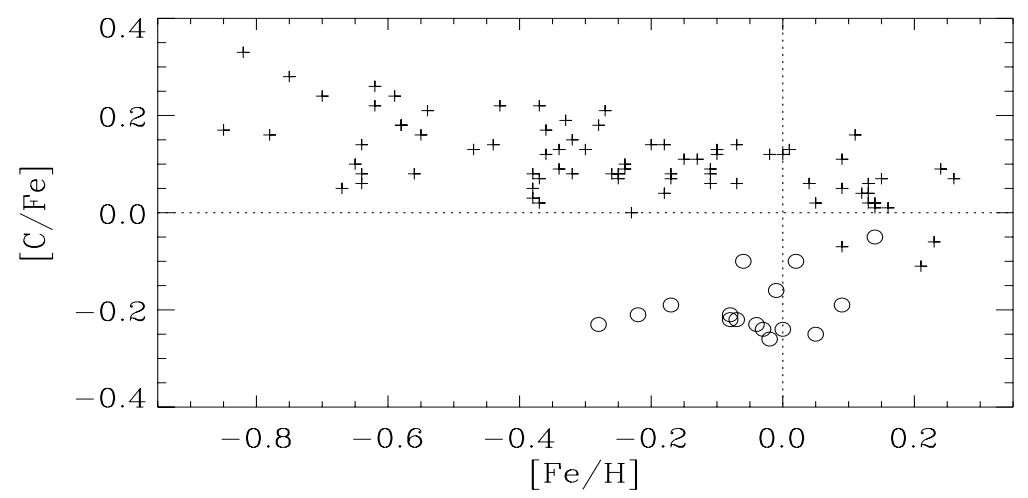

Figure 1. $[\mathrm{C} / \mathrm{Fe}]$ as a function of $[\mathrm{Fe} / \mathrm{H}]$. Abundance ratios for the Galactic field red clump stars are indicated by circles, for field dwarf stars - by 'plus' signs (Gustafsson et al. 1999).

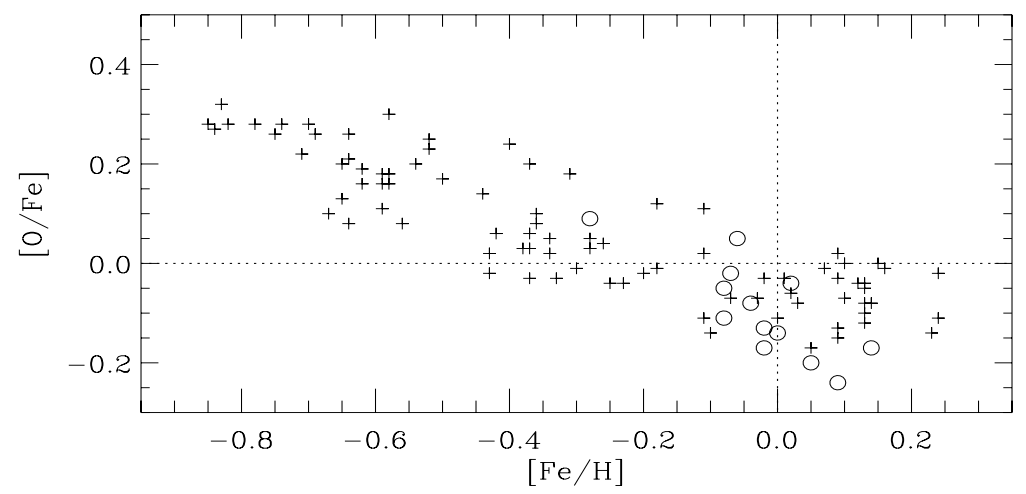

Figure 2. $[\mathrm{O} / \mathrm{Fe}]$ as a function of $[\mathrm{Fe} / \mathrm{H}]$. Abundance ratios for the Galactic field red clump stars are indicated by circles, for field dwarf stars - by 'plus' signs (Edvardsson et al. 1993).

\section{Observations and method of analysis}

The spectra for 17 stars, selected from the Hipparcos Catalog (Perryman 1997), were obtained on the Nordic Optical Telescope (La Palma) with the SOFIN échelle spectrograph. The 2 nd optical camera $(R \approx 60,000)$ was used to observe simultaneously 13 spectral orders, each of $40-60 \AA$ in length, located from $5650 \AA$ to $8130 \AA$.

The spectra were analyzed using a differential model atmosphere technique. The program packages, developed at the Uppsala Astronomical Observatory, were used to carry out the calculations of theoretical equivalent widths of lines, synthetic spectra and a set of plane parallel, line-blanketed, flux constant LTE model atmospheres.

The effective temperatures for the stars were determined using the $(B-V)_{o}$ and $(b-y)_{o}$ color indices and the temperature calibrations by Alonso et al. (2001). All the effective temperatures were carefully checked and corrected if needed in order to achieve the LTE excitation balance in the iron abundance results. The gravities were found by forcing Fe I and Fe II to yield the same iron abundances. The microturbulent velocities were determined by forcing Fe I line abundances to be independent of the equivalent width.

Abundances of carbon were studied using the $\mathrm{C}_{2}$ Swan $(0,1)$ band head at $5635.5 \AA$. The wavelength interval 7980-8130 $\AA$ with strong CN features was analyzed in order to determine nitrogen abundances and ${ }^{12} \mathrm{C} /{ }^{13} \mathrm{C}$ isotope ratios. The oxygen abundances were determined from the $[\mathrm{O} \mathrm{I}]$ line at $6300 \AA$. For more details on the method of analysis and atomic data see Tautvaišienè et al. (2005b). 

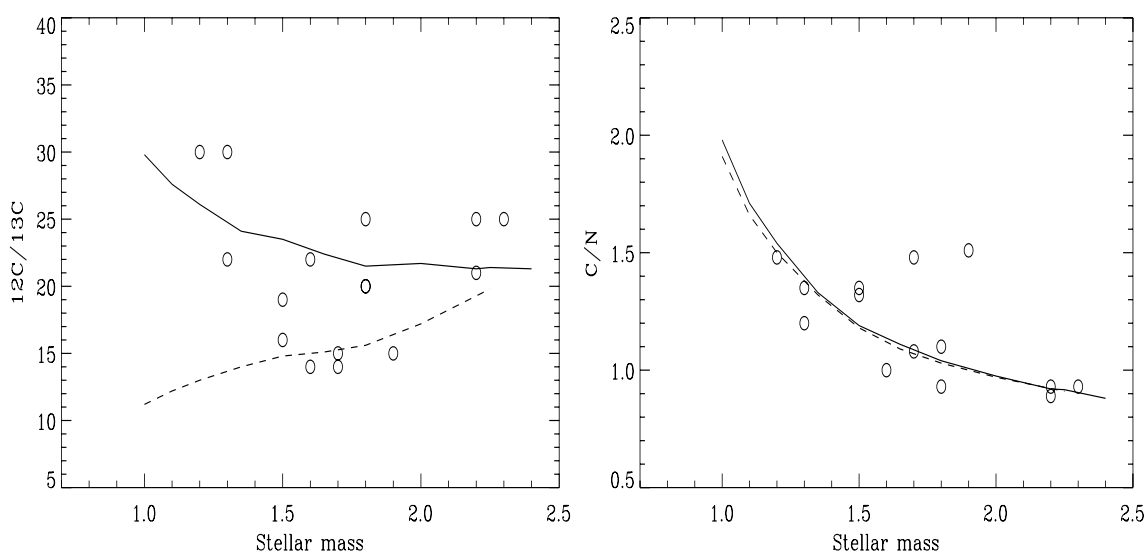

Figure 3. ${ }^{12} \mathrm{C} /{ }^{13} \mathrm{C}$ ratios (left) and $\mathrm{C} / \mathrm{N}$ ratios as a function of stellar mass. Results of the Galactic field red clump stars are indicated by circles. The theoretical models of the $1^{\text {st }}$ dredge-up (solid curve) and extra mixing (dashed curve) are taken from Boothroyd \& Sackmann (1999).

\section{3. $\mathrm{C}, \mathrm{N}$ and $\mathrm{O}$ abundances}

In comparison to the field dwarfs (Fig. 1), the results show that $[\mathrm{C} / \mathrm{Fe}]$ in the clump stars investigated is lowered by about 0.2 dex and $[\mathrm{O} / \mathrm{Fe}]$ is not altered (Fig. 2).

In the ${ }^{12} \mathrm{C} /{ }^{13} \mathrm{C}$ vs. mass plot (Fig. 3), the stars separate into two groups, one of which follows the trend of $1^{\text {st }}$ dredge-up model with ${ }^{12} \mathrm{C} /{ }^{13} \mathrm{C}$ ratios being in the range of $20-30$, depending on mass. The ${ }^{12} \mathrm{C} /{ }^{13} \mathrm{C}$ ratios of the other group are equal to about 15 and exhibit the extra-mixing in agreement with the model of Boothroyd \& Sackmann (1999).

The results of $\mathrm{C} / \mathrm{N}$ quite closely follow the $\mathrm{C} / \mathrm{N}$ vs. mass trend predicted by the $1^{\text {st }}$ dredge-up model (Fig. 3). The extra-mixing model by Boothroyd \& Sackmann (1999) does not predict extra $\mathrm{C} / \mathrm{N}$ changes to be noticeable at this metallicity and mass as well.

The work on $\mathrm{C}, \mathrm{N}$, and $\mathrm{O}$ abundances in clump stars is in progress. Further results will include the analysis of more than 30 other field clump stars.

\section{Acknowledgements}

This project has been supported by the European Commission through the "Access to Research Infrastructures Action" of the "Improving Human Potential Programme", awarded to the Instituto de Astrofísica de Canarias to fund European Astronomers' access to the European Nordern Observatory, in the Canary Islands.

\section{References}

Alonso, A., Arribas, S. \& Martínez-Roger, C. 1999, A\&AS, 140, 261

Alonso, A., Arribas, S. \& Martínez-Roger, C. 2001, A\&A, 376, 1039

Boothroyd, A. I. \& Sackmann, I.-J. 1999, ApJ, 510, 232

Edvardsson, B., Andersen, J., Gustafsson, B., Lambert, D.L., Nissen, P.E., \& Tomkin, J. 1993, $A \mathscr{E} A, 275,101$

Gustafsson, B., Karlsson, T., Olsson, E., Edvardsson, B., \& Ryde, N. 1999, A\&A, 342, 426

McWilliam, A. 1990, ApJS, 74, 1075

McWilliam, A. \& Rich R. M. 1997, ApJS, 91, 749

Perryman, M.A.C. 1997, ESA SP, 404, 231

Tautvaišienė, G., Stasiukaitis, E., Puzeras, E., Gray, D. F., \& Ilyin, I. 2005a, ESA SP, eds. F. Favata, G. Hussain, \& B. Battrick, 560, 989

Tautvaišienè, G., Edvardsson, B., Puzeras, E., \& Ilyin, I. 2005b, A\& A, 431, 933

Zhao, G., Qiu, H.M., \& Mao, S. 2001, ApJ, 551, L85 